\title{
Comment on "Designing Robust N-of-1 Studies for Precision Medicine: Simulation Study and Design Recommendations"
}

Reid D Landes, $\mathrm{PhD}$

Department of Biostatistics, University of Arkansas for Medical Sciences, Little Rock, AR, United States

Corresponding Author:

Reid D Landes, PhD

Department of Biostatistics

University of Arkansas for Medical Sciences

4301 W Markham St

Slot 781

Little Rock, AR, 72205

United States

Phone: 15015266714

Email: rdlandes@uams.edu

Related Article:

Comment on: https://www.jmir.org/2019/4/e12641/

(J Med Internet Res 2020;22(9):e16179) doi: 10.2196/16179

\section{KEYWORDS}

sample size; misleading statements

In "Designing robust N-of-1 studies for precision medicine: Simulation study and design recommendations" by Percha et al [1], the authors use misleading language when speaking about the required numbers of samples regarding results in Figure 4a. For example, they write on page 8:

In Figure 4a, we see that for effect sizes of 0.1, 0.2, and 0.3, more than 100 samples are needed to obtain a power of 0.8 (at a standard 5\% significance level). For an effect size of 0.4, at least 100 samples are needed. For effect sizes of 0.5, 0.6, 0.7, 0.8, 0.9, and 1.0, the numbers of samples needed to attain a power of 0.8 are approximately $65,45,35,26,21$, and 18 , respectively. [Figure 4]

Since Figure $4 \mathrm{a}$ is exactly equivalent to power curves from a two-sample, equal-variance $t$ test (see Figure 1; generating $\mathrm{R}$ code provided in Textbox 1), the numbers of samples are for one of the two treatments; thus, the total numbers of samples are doubled. An easy fix in most instances of the unclear language is to add "per treatment" after "samples." I provide a list of potential clarifying edits to the article's text below (but may have missed some instances):
- $\quad$ Figure 4c caption: "(ie, number of samples per treatment, with sampling rate fixed at 1 sample per time unit)"

- $\quad$ Figure 4a and 4b: the label for the horizontal axis should be "Number of samples per treatment"

- $\quad$ Page 8: "In Figure 4a, we see that for effect sizes of 0.1 , 0.2 , and 0.3 , more than 100 samples per treatment are needed to obtain a power of 0.8 (at a standard 5\% significance level). For an effect size of 0.4 , at least 100 samples per treatment are needed. For effect sizes of 0.5 , $0.6,0.7,0.8,0.9$, and 1.0, the numbers of samples per treatment needed to attain a power of 0.8 are approximately $65,45,35,26,21$, and 18, respectively."

- $\quad$ Page 9: "For an effect size of 0.5 and $\sigma_{\mathrm{p}}=0.0,0.4,0.8,1.2$, $1.6,2.0$, the numbers of samples per treatment needed to obtain a power of 0.8 are $61,76,89,111,135$, and 176 , respectively. For an effect size of 1.0 , the numbers of samples per treatment needed are 20, 24, 28, 34, 43, and 53, respectively."

- Page 9: "For $\sigma_{\mathrm{p}}=0.0,0.4,0.8,1.2,1.6,2.0$ and $\alpha=0.1$, the numbers of samples per treatment required are $36,64,110$, 174,228 , and 250, respectively. For $\alpha=10.0$, the numbers of samples per treatment required are only 20, 23, 28, 34, 42 , and 53, respectively." 
Figure 1. For effect sizes ranging from 0.1 to 1.0, power of a 0.05 level two-sample $t$ test plotted by $n$, the number of samples in one treatment group. Total sample size is assumed to be $2 n$.

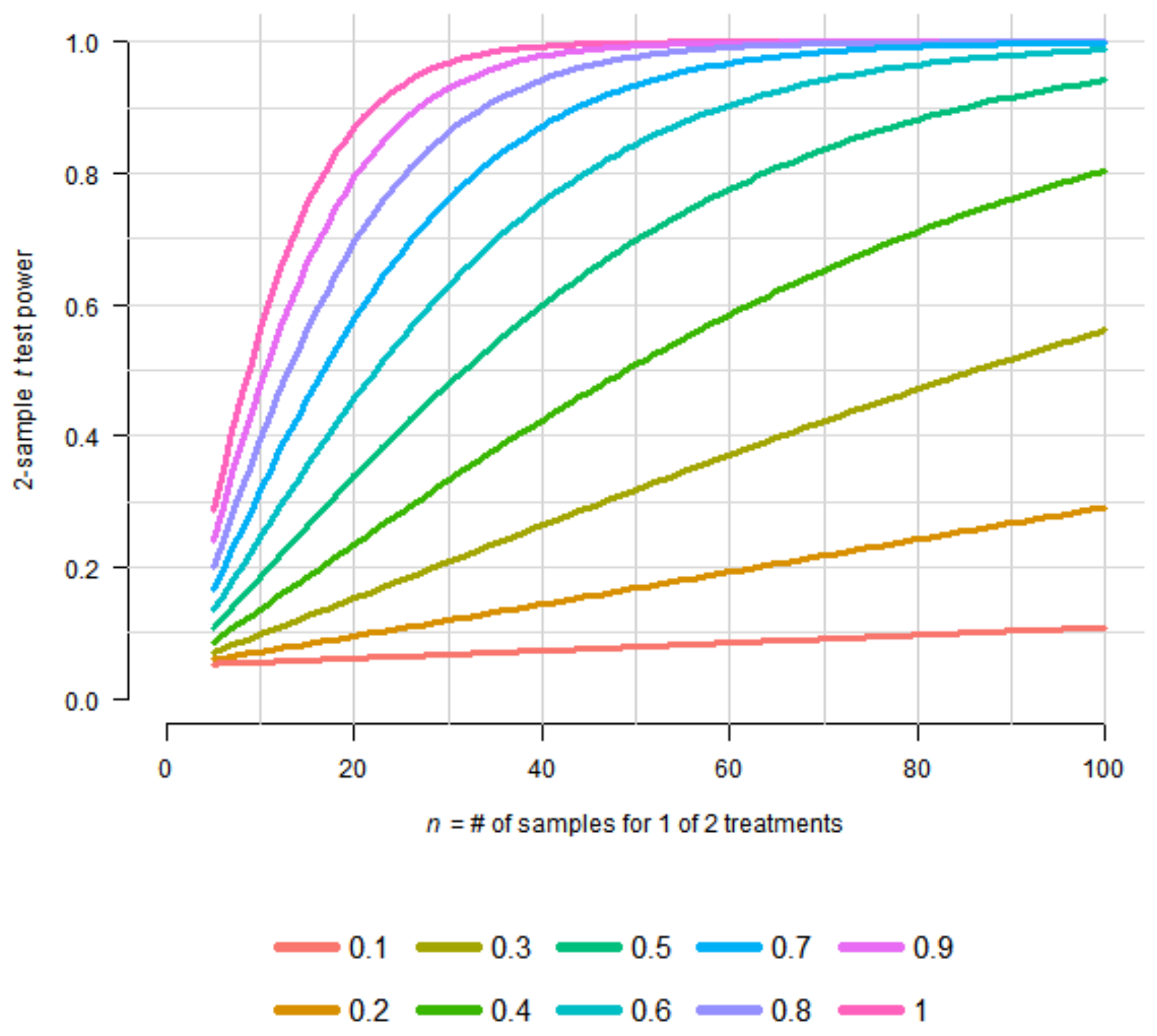

Effect Size 
Textbox 1. $R$ code.

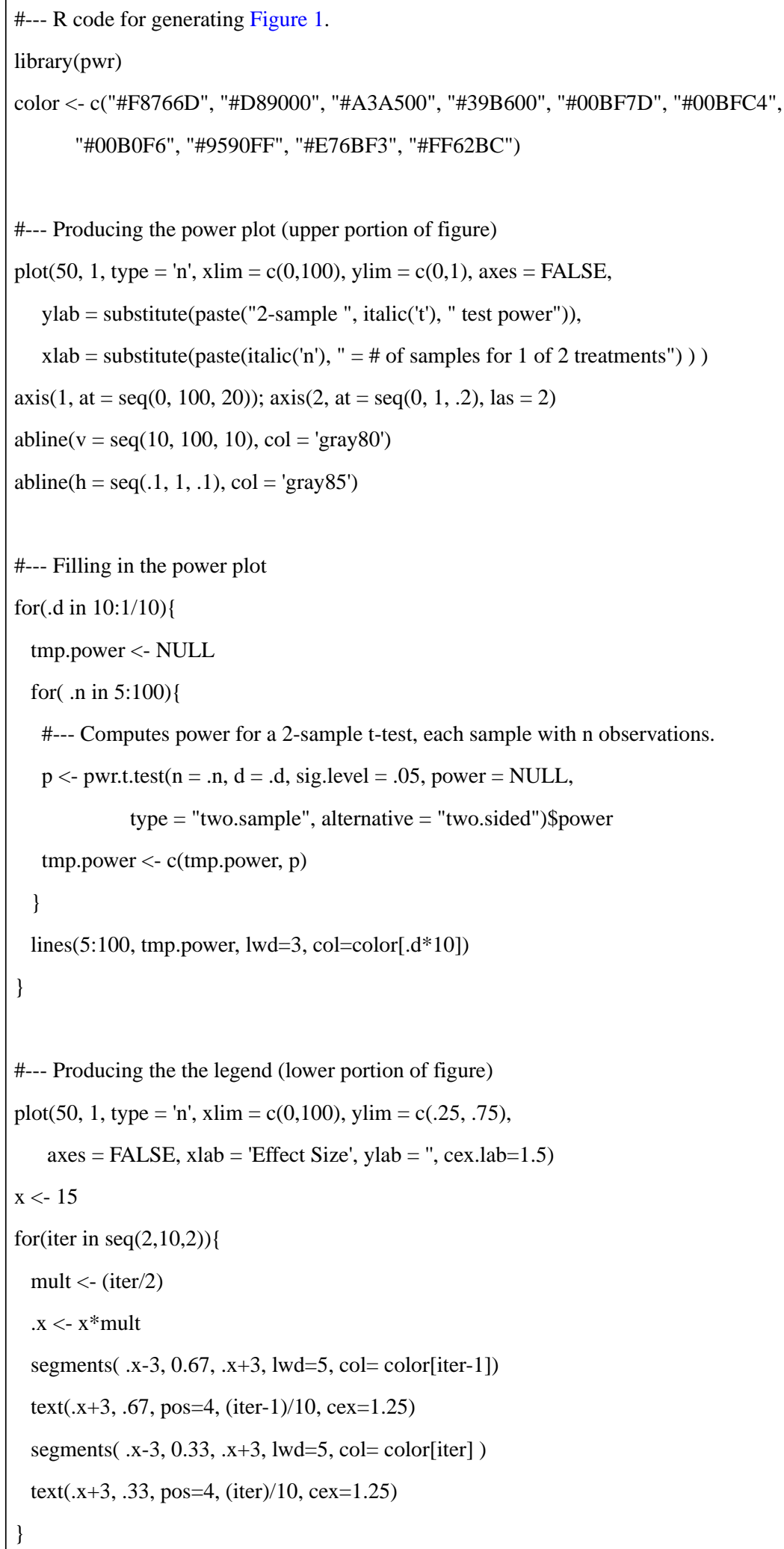

\section{Editorial Notice}

Percha and colleagues have agreed to the above changes; these changes have been made to the original paper.

\section{Conflicts of Interest}

None declared. 


\section{Reference}

1. Percha B, Baskerville EB, Johnson M, Dudley JT, Zimmerman N. Designing Robust N-of-1 Studies for Precision Medicine: Simulation Study and Design Recommendations. J Med Internet Res 2019 Apr 01;21(4):e12641 [FREE Full text] [doi: 10.2196/12641] [Medline: 30932871]

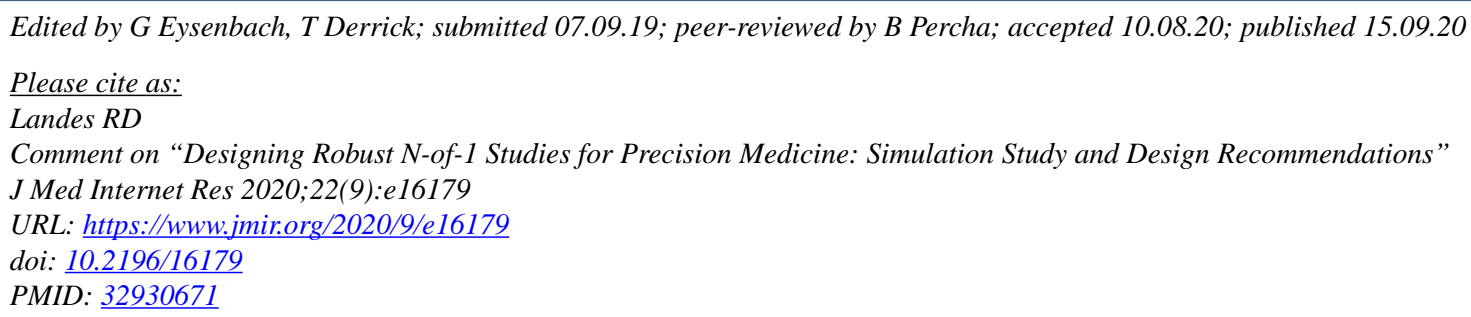

CReid D Landes. Originally published in the Journal of Medical Internet Research (http://www.jmir.org), 15.09.2020. This is an open-access article distributed under the terms of the Creative Commons Attribution License (https://creativecommons.org/licenses/by/4.0/), which permits unrestricted use, distribution, and reproduction in any medium, provided the original work, first published in the Journal of Medical Internet Research, is properly cited. The complete bibliographic information, a link to the original publication on http://www.jmir.org/, as well as this copyright and license information must be included. 